\title{
On the adaptation of CAN BUS network for use in the ship electronic systems
}

\author{
Andrzej Piętak, Assoc. Prof. \\ Maciej Mikulski, M. Sc. \\ University of Warmia and Mazuria in Olsztyn
}

\begin{abstract}
This paper presents an analysis of application of the CAN - based marine electronics standard NMEA 2000, recently developed one, on board sea-going vessels, especially in the context of small, recreational, sailing and motor craft. The idea and development of the system has been briefly presented. The properties of the CAN network has been described and the use of its functions by the NMEA system, discussed. The discussion has been performed for all layers of the communication protocol, according to the Open Systems Interconnection Reference Model worked out by ISO. All the features have been summarized and analyzed in the context of their possible use in various shipboard systems.
\end{abstract}

Keywords: controlled area network; CAN; CAN BUS; linear network communication; NMEA2000 standard; NMEA 2000 network

\section{INTRODUCTION}

In the beginning phase of ship electronics development, each device was an entirely independent unit. Devices such as electronic log, echo sounder or gyrocompass were carrying out their own tasks, being not connected to other instruments. With the development of new technologies it soon became clear that it is crucial for the separate devices to communicate with each other. Introduction of autopilot required to get a signal from gyrocompass and so on. Some producers have introduced their own devices to connect the systems [1,2]. With the development of digital techniques the need of standardization of the communication protocol used by the navigational devices became obvious. The breakthrough came in the year 1980 when the NMEA (National Marine Electronics Association) introduced the NMEA0180 standard. For the following years the standard was being improved. The NMEA0183 standard introduced in 1983 incorporated radical changes in the system which enabled the mutual communication between virtually each device of a vessel's electronics, and also easy exchange of data with PC computer. The NMEA0183 standard, with the passage of years, has undergone significant evolution, and operation of new devices has been added. Towards the end of the $20^{\text {th }}$ century the contemporary standard turned out to be insufficient. The NMEA0183, in principle, provides the linear data transfer only from the transmitter to several receiving devices (the so-called 'listeners'). The progressing strong integration of the on-board systems results in that the modern maritime electronic equipment requires data coming from many sources. Without a network standard which would enable such integration of data, the equipment producers have to provide several inputs or introduce such solutions which cumulate data from several transmitting devices into one channel. The first solution is connected with a higher cost and additional complication in wiring system. On the other hand the NMEA0186 protocol operating with the speed of $4800 \mathrm{bit} / \mathrm{s}$, is able to provide approximately 10 sentences per second, which is absolutely sufficient when one device transmits, but it fails when the system starts to join the data.

The new standard of the linear network communication - NMEA2000 worked out in 2001 by the National Marine Electronics Association, in which the integrated circuits of the CAN network (Controlled Area Network) is used, was the answer to the growing expectations dealing with joining and exchange of data among the electronic ship devices. The CAN has been originally worked out for the car industry but due to its easy access and low price of the components, is now currently applied in many industrial branches. The standard is becoming more and more popular. The reputable producers of navigational equipment have started to introduce products ready to cooperate with the NMEA2000 network, onto the market. The first applications of the standard have appeared not only in the elaborate on-board systems of big vessels but also in the projects of navigational and propulsion systems of small recreational crafts. Despite the growing interest of the marine equipment producers, the knowledge concerning the new system seems to be not wide- spread especially as far as its use in the yacht industry has been concerned. Also the literature on the standard in question as well as on the possibilities of using the well-known CAN main technology in the electronic systems of small vessels is very scarce. Thus, it seems reasonable to provide a wider commentary on both the systems (i.e. the NMEA2000 and CAN BUS) and to research the legitimacy of using them, especially in the aspect of recreational motor and sailing vessels. 


\section{THE NMEA2000 STANDARD}

The NMEA 2000 is a cheap linear communication network which operates with the speed of $250 \mathrm{kbit} / \mathrm{s}$ using the integrated circuits of the CAN network. The main difference between the NMEA 2000, and the earlier standard, NMEA 0183, apart from the transmission speed (of $4.7 \mathrm{kbit} / \mathrm{s}$ for NMEA 0183), is that the first constitutes the whole network of data exchange, and the second one is only the communication protocol. The NMEA 2000 network, unlike preceding standards, enables connecting many electronic devices through one communication channel in which several devices may transmit the data simultaneously. Like the NMEA 0183, the NMEA 2000 introduces standard definitions and formats of a message, but in addition, it provides broader terms of managing the network, device identification, sending the commands and receiving the data. Just as in the case of any network communication, joint between the central units takes place through the solitary lead, which considerably simplifies the installation and lowers its cost in the case of more elaborated systems. In the system, data are transferred in the form of data frames with reserved bits in order to identify and detect errors and confirm the receiving of a message. Each frame consists of 8-byte data space and 29-byte identification field which determines the priority of a message and identifies the broadcasting and receiving element in the network. As only a small part of the frame is allotted to the data, the standard is mainly intended for the transmission of short messages but, owing to the advanced error control, with a high level of reliability. Messages may be transmitted periodically, sent only in given circumstances or at the request of given receivers.

In accordance with the Open Systems Interconnection Reference Model (OSI model) worked out by ISO, [3], the NMEA 2000 standard defines all the layers (the OSI model is based on the seven-layer organization of the communication protocol) essential for proper operation of a network standard, in accordance with the taken-up assumptions. The most important components of the NMEA 2000 network are presented below.

Tab. 1. Elements of the NMEA 2000 standard, with the differentiation into layers in accordance with the ISO/OSI model, based on [3], [4].

\begin{tabular}{|c|c|}
\hline Physical Layer & $\begin{array}{c}\text { Fully defined in the standard, } \\
\text { it normalizes the sort of } \\
\text { cables and connectors, the } \\
\text { characteristics of signals etc. }\end{array}$ \\
\hline Data Layer & $\begin{array}{c}\text { Defined by the ISO 11783-3 } \\
\text { standard with the additional } \\
\text { requirements contained in the } \\
\text { NMEA 2000 standard. }\end{array}$ \\
\hline Network Layer & $\begin{array}{c}\text { Currently non-defined in the } \\
\text { NMA 2000, it will be defined } \\
\text { in future versions of the } \\
\text { standard. }\end{array}$ \\
\hline $\begin{array}{c}\text { Network management } \\
\text { includes the transport, } \\
\text { session and presentation } \\
\text { layers in accordance } \\
\text { with the OSI model) }\end{array}$ & $\begin{array}{c}\text { Defined in the ISO 11783-5 } \\
\text { standard with the additional } \\
\text { requirements contained in the } \\
\text { NMEA 2000 standard. }\end{array}$ \\
\hline $\begin{array}{c}\text { Application Layer } \\
\text { Fully defined in the NMEA } \\
2000 \text { standard }\end{array}$ \\
\hline
\end{tabular}

The main characteristics of the NMEA 2000 network, which result from the accepted standards and definition, are contained in Tab. 2.

Tab. 2. Characteristics of the network in accordance with [5]

\begin{tabular}{|c|c|}
\hline \multicolumn{2}{|r|}{ Characteristics of the network } \\
\hline Architecture & $\begin{array}{l}\text { - Wiring of a main by using the twisted- } \\
\text { pair cable, intended for the supply, data } \\
\text { transfer and functioning of interface. } \\
\text { Linear network with a single backbone } \\
\text { cable, separate central units joined to } \\
\text { the main through the short cables of } \\
\text { the AUI interface. }\end{array}$ \\
\hline Functioning & $\begin{array}{l}\text { Access to the network: carrier sense } \\
\text { / multi-access / access arbitration } \\
\text { determined by the CAN network } \\
\text { controller. } \\
\text { - Network of a multi-master type (lack } \\
\text { of a central control unit). } \\
\text { - Self-configuring. } \\
\text { - Special network tools necessary for the } \\
\text { diagnostic purposes, but not essential } \\
\text { for the functioning of a network. }\end{array}$ \\
\hline Size & $\begin{array}{l}\text { - Physical central units: } 50 \text { connections } \\
\text { at max. } \\
\text { - Functional central units: } 252 \mathrm{IP} \\
\text { addresses at max. } \\
\text { - Length of a network: up to } 200 \mathrm{~m} \text { (at } \\
\text { the } 250 \mathrm{kbit} / \mathrm{s} \text { speed of transfer). }\end{array}$ \\
\hline
\end{tabular}

\section{THE CAN BUS - THE BASIS FOR THE PHYSICAL LAYER OF THE NMEA 2000}

The physical layer defines mechanical and electrical aspects of connection between the elements of network. The mechanical part of physical layer (kind of cables and the mean joining them) of a network is fully described by the NMEA 2000 standard and is not a subject of this work. Characteristics of the electric physical layer can be covered by the following points:

- Access to media is controlled by the CAN standard defined as in the ISO 11898 standard.

- The CAN uses dominative/recessive bit transmission.

- Time delay and capacity of a network limits the frequency of bit transfer and the length of a backbone cable.

- LVDS method significantly improves the resistance to interferences of transmission.

- The network single-point common signal reference controls ground voltage levels and reduces the RFI.

The CAN network has been worked out in the second half of the 1980s by the Bosch Company. After a number of changes the CAN network specification present in the ISO 1898 standard, has become the international standard to be used in the vehicles. The controllers of the network, owing to its extensive use in the automotive industry, are available at a price lower than most of the ASIC networks used to control the mains of measurement-control systems (such as e.g. Profibus). Thus, the CAN is widely used also in industrial automation systems as a main for the communication with sensors and actuators. Many advantages of the standard have led to its use also in the vessel networks. 
The CAN network is a linear, bit-orientated main, to which the CSMA/CA method of the access to the main avoiding the collisions and errors of transmission, is applied. It requires the time of reaction of all drivers to be no longer than the time of transfer of one bit. It imposes significant limitations on the length of a main which has to fulfill the following condition:

$$
\mathrm{L} \leq \mathrm{C}_{1} \cdot \frac{1}{\mathrm{~K}}
$$

where:

$$
\begin{aligned}
& \mathrm{L}-\text { ength of a main [m] } \\
& \mathrm{C}_{1}-\text { proportionality constant (of the value varying } \\
& \mathrm{K}-\text { between } 40 \text { and } 50 \text {, acc. different sources) } \\
& \mathrm{T} \text { transmission speed [Mbit/s]. }
\end{aligned}
$$

The length of the main has to be the smaller the bigger the speed of transmission, as all the CSMA/CA devices on the main have to work with the same speed, and as in the transmission line - in case of long lines - the differences of a signal, resulting from delays in the transmitter/receiver or amplifier, may appear. The relation described in Eq. (3.1) is an empirical formula. In the NMEA 2000 standard the CAN network transmission speed has been set to $250 \mathrm{kbit} / \mathrm{s}$ which is equivalent to 200 - meter long main cable.

The quick transmission method through copper cables which have low amplitude, power and small interferences (LVDS), is based on the fact that the CAN main has to be closed at both ends with conditioning resistors (so called terminators) of a value depending on cable impedance. The transmitter transmits small electric current which depends on the logical value (usually of 3,5 mA ) which is to be transmitted through one cable. After going through the final resistor, the signal comes back in the opposite direction. The receiver measures the difference of voltages in both cables in relation to the joint reference signal in order to determine the logic state. The arrangement of the typical network based on the CAN main is presented in Fig. 1. The small signal amplitude and strong coupling of the electric and magnetic field between the pair of cables results in that the emission of electrical noise (and the loss of energy) is very low.

ECU 1

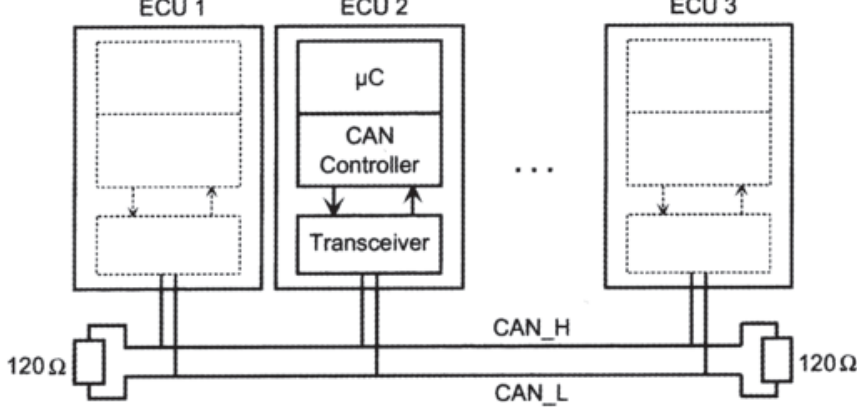

Fig. 1. The arrangement of the typical network based on the CAN main [7]

The LVDS demands powered network interfaces and joint reference signal to all the central units of the network. Two signal lines transmit a differential signal measured in relation to the reference signal. In the CAN networks two logical levels which complement each other, are present, namely: 'dominant' or 'recessive'. During the simultaneous transmission of a 'dominant' and 'recessive' bit through two different devices the resulting 'dominant' level is acquired. Contractually, the 'dominant' level is marked " 0 ", and the 'recessive' level " 1 ". The level " 0 " has lower priority than the level " 1 ". The ISO 11898 standard defines the level of individual states as follows:
- Dominant state:

CAN_H $=3,5 \mathrm{~V}$, CAN_L $=1.5 \mathrm{~V}$, $\mathrm{V}_{\text {diff }}={ }_{\text {CAN_H }}-\mathrm{CAN}_{-}{ }_{-} \mathrm{L}=2.0 \mathrm{~V}$

- Recessive state:

$$
\text { CAN_H }=2,5 \mathrm{~V}, \text { CAN_L }=2.5 \mathrm{~V} \text {, }
$$$$
\mathrm{V}_{\text {diff }}={ }^{-} \text {CAN_H }-\mathrm{CAN}_{-}^{-} \mathrm{L}=0.0 \mathrm{~V}
$$

The low differential voltage occurring in a system consumes very small amount of energy in comparison to other solutions, which is especially important in case of sailing vessels. The standard also enables the using of typical $12 \mathrm{~V}$ yacht batteries for the powering of the whole network, in the case when length of a backbone cable and number of central units is sufficiently small. For bigger installations several power sources can be used. Power can be delivered through the main cable or the separate lead (dedicated twisted - pair cable). Such option enables additional power consumption by the device with minimum interface complication. In both the cases the power supply and the reference signal for the interface circuits cannot have direct connection to the power supply circuit or the device grounding in the network. The isolation can be realized in various ways. The most popular one is the using of the optic isolation in the device between the CAN network controller and other circuits. Such isolation which complies with the ISO 11898 standard requirements, is presented in Fig. 2.

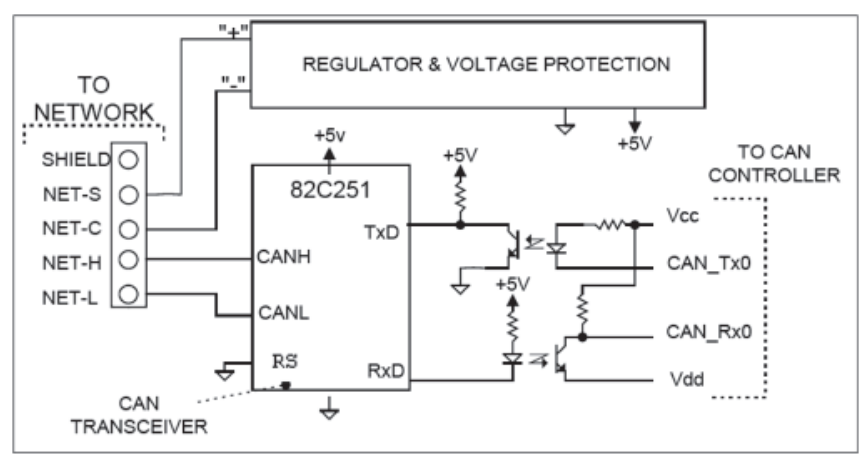

Typical Isolated Network Interface

Fig. 2. The example of the optic isolation between the circuits of the interface and devices in the network in accordance with the ISO 11898 standard [6]

\section{THE CAN IN THE DATA LAYER}

Like in the case of physical layer, some features of the data connection layer are determined by choice of the CAN as the main of network. The NMEA 2000 fully makes use of the international standard of the ISO 11783-3 data connection layer, which is virtually identical with the SAE J1939-21 standard (i.e. the specification of the CAN for the lorries, delivery trucks and trailers). Additional requirements contained in the NMEA 2000 ensure better copying a system with special types of data and formats transmitted through a navigational device and support special construction of such devices.

In the data connection layer of the NMEA 2000 network, main functions of the CAN interface are: generating the linear stream of bits, controlling the access to the network as well as controlling errors and automatic transmission of messages in which the errors occurred.

The CAN is a broadcast system in which every sender transmits his own messages. Each driver in the network receives messages and, on the basis of a message identifier, decides whether the data will be processed or ignored. In the current specification of the CAN, are incorporated, a. o., 


\begin{tabular}{|c|c|c|c|c|c|}
\hline $\begin{array}{c}\text { Start } \\
\text { Bit }\end{array}$ & $\begin{array}{l}\text { Eontrol } \\
\text { Bits }\end{array}$ & & & & $\begin{array}{l}\text { Bus } \\
\text { Idle }\end{array}$ \\
\hline $\begin{array}{c}11 \text { or } 29 \text { bit } \\
\text { Message Identifier }\end{array}$ & 7 bit & Data 0 ... 8 Byte & $\begin{array}{l}15 \text { bit } \\
\text { CRC }\end{array}$ & \begin{tabular}{|c|} 
Acknowledge \\
\& End of Frame
\end{tabular} & \\
\hline
\end{tabular}

Fig. 3. The CAN communication format (without the separating bits)

29-bits field of identification, the address of a transmitter (specific or global address), type of transmitted data and information about priority. When collision between two devices trying to send data through the main takes place, the CAN compares bit by bit the sent data and this way decides about giving the access, at the same time the arbitration is based on the priority of a message. This method called the Carrier Sense Multiple Access with Collision Avoidance + Arbitration on Message Priority (CSMA/CA + AMP) has such advantage over the standard CSMA/CA protocol that it does not generate time losses.

For error handling and control, each frame contains the so-called cyclic redundancy check (CRC) owing to which the receivers synchronize their execute signal generators with the transmitter. Transmitter, after checking the format and data frame $\mathrm{CRC}$, sends confirmation of receiving the communication by using the ACK bit (Acknowledge) and the end of frame bits. In case of detecting an error by any of the controllers of the network, it sends the application of the error, which results in ignoring the incorrect communication by all the receivers. Owing to this fact the conformity of data in the entire network is guaranteed. The transmitter which has received the error application, immediately and automatically transmits the message in which an error occurred. The CAN detects if a given central unit generates repeating errors and automatically disconnects it from the network to protect the rest of the elements. Results of the research [7] indicate that the error rate of transmission in the CAN system is smaller than $10^{-11}$ (i.e. percentage of undetectable errors).

The NMEA 2000 standard fully makes use of the described functionality of the CAN network, and in the same time the main tasks it imposes on it in the data connection layer, are the following:

- ensuring the method of transmitting the data which are too big to be contained in a single 8-bit data frame

- defining the set of messages in order to issue a command to the network devices (for example: at the data request or the confirmation request).

The CAN has been originally designed as a transmission network between the sensors/detectors, actuators and the steering system. Such communication does not require the transmission of excessively elaborated messages hence the 8-bit frame was deemed fully sufficient. Due to the specificity of navigational devices, many messages in the NMEA 2000 system exceed the capacity of the standard CAN data controller. In order to deal with this problem, the fast-packet method which enables the transmission of 223 bytes with one identifier, is used for the transmission. This method is about transmitting the frame sequence, the first of which contains the data concerning size of a packet, sequence counter which serves as means for differentiating the messages of the same size in a package and the frame counter. Each additional frame uses one byte of its data space for sequence and frame counters.

The NMEA 2000 expands the capabilities provided by the ISO 11783-3 standard, providing the new type of a messagecommand, unprecedented in the CAN mains adaptations, which enables setting the specific value or inducing concrete actions in the receiving device. In addition, the message-command defined in the NMEA 2000, enables setting the time interval of the transmission of concrete data in the transmitting device. For example, a message containing way-points usually consists of the position, name, number and symbol of this point. By using such request the device can force transmitting information concerning certain 'way-point' by providing its name, number or position.

\section{OTHER LAYERS OF THE NMEA 2000 NETWORK}

The procedures used in the managing of the NMEA 2000 network result directly from the ISO 11783-5 standard ( based on the SAE J1939-81). According to the guidelines the role of this layer can be simply described as: assigning the addresses and identification of devices in the network, as well as initiation of a system after switching-on the power. From among the 256 IP addresses available in the NMEA 2000, only those from 0 to 251 can be assigned to the devices. The set of 255 addresses is the global address used to send messages to all the devices and because of the character of the network (broadcast network) it is the address mostly used while sending messages. Other addresses are reserved to report errors or to be used in next versions of the standard. On the contrary to current CAN applications in accordance with the NMEA 2000, all the devices have to be self-configuring and able to automatically acquire the address while switching-on the system (as devices do not have permanent addresses). The procedure of assigning addresses makes use of the content of the messages data field-command of the address sent by each device during the start. The message contains the name of a device, constituted from the numbers identifying its type and producer and the unique number describing its concrete copy. Assigning the address takes place according to the priority of name. Message-command defined in the data layer of the NMEA 2000 network, can be used to detect information concerning devices connected to the network, to build the map of whole system available to the user. The ability of inserting installation comments by the performer, which can be transmitted at the request, is the additional functionality of the network. The application layer defines composition of all messages which are to be sent in the network. All messages are organized in the parameter groups which are identified by the parameter group number (PGN number), contained in the CAN frame identification field.

\section{ANALYSIS OF THE SCOPE OF APPLICATIONS OF THE CAN TO VESSEL NETWORKS}

In the field of vessel systems, their progressing integration takes place. The phenomenon occurs to a different degree on board merchant ships, fishing vessels and yachts. However, irrespective of a purpose, size and complexity level of installed subsystems, to introduce standardization of the process of data exchange between vessel's separate devices, is necessary. The general configuration of a vessel system is illustrated in Fig. 4. 


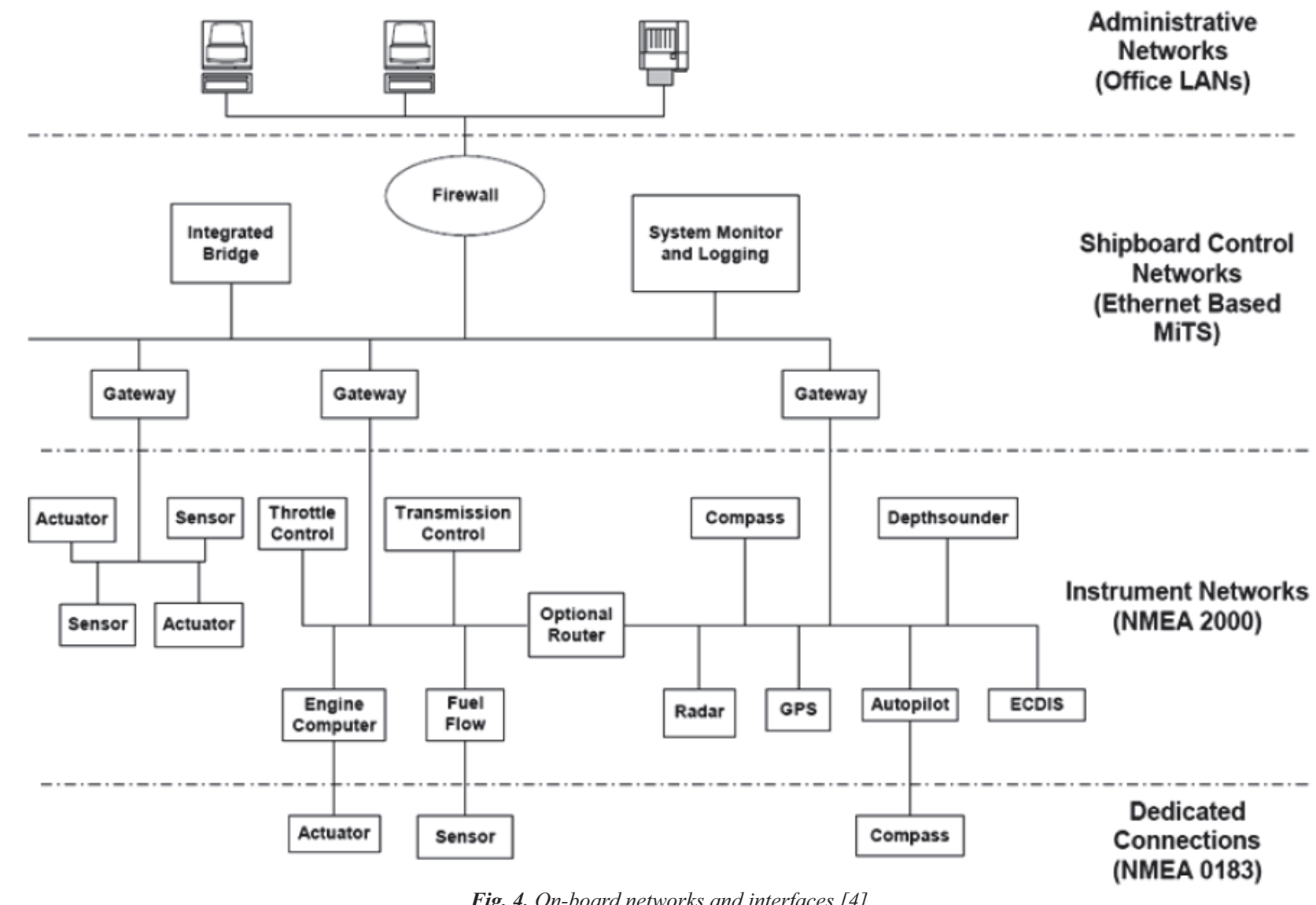

Big merchant vessels, due to their specificity, have the elaborate system of administrative data such as a.o. cargo amount and stowing place. Such databases often have to be accessed not only from the terminals located in different parts of vessel, but also via satellite systems (for example the IMMARSAT C), coupled with the central server in the shipowner's office. However, the administration system is important as far as the effective managing of the vessel is concerned, its efficient work does not significantly influence safety of navigation. Therefore it is not included in the detailed regulations and, out of concern for the users comfort, it was virtually taken over by the PC computers working in the standard LAN network based on the commonly available subsystems.

Decisions which directly influence safety of a vessel and her crew as well as those concerning the ecological safety of greater and greater importance, are taken mostly on the bridge or other control centres being a part of a bridge integrated system. In order to take up such decisions effectively and to guarantee their efficient implementation, captain and officers need the access to data coming from many vessel's systems (of navigation, engine room and fire control etc). The sea transport specificity and special demands concerning reliability eliminate the use of traditional LAN network in such systems despite the requirements concerning the type and speed of data transmission are similar. Big amount of data which has to be sent through the decision support system in the same time disqualifies the CAN network which is dedicated to transmit short messages with high reliability. Requirements concerning the scope of communication between the subsystems of integrated bridge were regulated by the Norwegian Maritime Information Technology Standard (MiTS) [8]. The system based on the TCP/IP protocol (Ethernet network) is currently in the phase of tests and implementation. The surge of interest in Ethernet-based systems in the management layer, is expected [5]. It is also confirmed by the recent application of

the MiTS standard as a basis for the European IEC 61162-4 standard.

However, it appears that systems based on the TCP/IP protocol, propagated by the MiTS, do not fully fit all the vessel communication levels. Separate vessel's subsystems such as engine control or navigational system usually perform strictly defined functions and often regulate processes with the time constant of the order of milliseconds. Therefore they require a real-time data exchange system. The CAN is such system and, on the contrary to Ethernet networks, it is of deterministic kind. Moreover, engine or navigation control, and other control subsystems are usually more autonomic and they transfer relatively small amounts of data (short communications) in relation to other vessel systems. The network dedicated to ensure communication between simple actuators and sensors, i.e. relatively cheap elements, should be of a lower cost per central unit than other vessel networks. All the requirements result in that the choice of an inexpensive CAN network to the subsystem communication, is fully justified.

\section{AN EXAMPLE OF A NMEA 2000 - BASED SYSTEM DESIGNED FOR A MOTOR YACHT}

With the introduction of the standard in 2001, attempts to applying it have begun. The test installation has been made on a 25 -feet Cherokee class motor boat used by the U.S. Coast Guard. The project assumed the use of electronic throttle and shift controls which transmitted NMEA 2000 - format messages into twin onboard Mercruiser engines equipped with the Teleflex Marine controllers [12]. This way, any mechanical connection between the engine room and the helm station has been eliminated. Beginning from 2008, Yamaha, as the first company, introduced a stock - made motorboat with CAN -based electronic throttle control. In the same time, the largest 

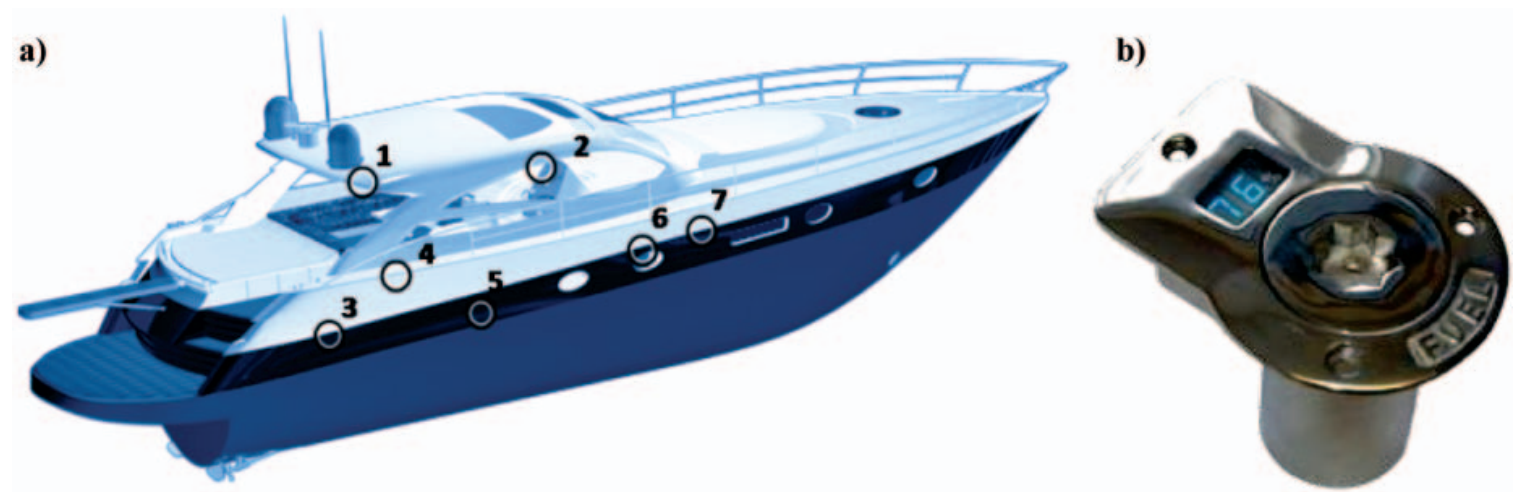

Fig. 5. a) ${ }^{1}$ Lay-out of the most important elements of the Albatross Control and Management System on a 60 - feet motor yacht. 1 - navigation system section, 2 - helm station with data visualization and sections control, 3 - engine room controller, 4 - energy management section, 5 - resources management, $\mathbf{6}$ - lighting and comfort system controllers, 7 - secondary monitoring station (by means of a multi-function display and wireless network).

b) A fuel filler with an electronic filling percentage indicator - an example of an active NMEA 2000 compatible element used on the yacht

maritime electronics manufacturers begun to develop new navigation equipment meeting the requirements of the NMEA 2000 protocol [9], [10].

In the present time, boat yards have a possibility of using full functionality of the new network solution. Examination of yacht systems that are entirely based on CAN protocol, seems most interesting. In Fig. 5 is presented a 60 - feet motor yacht equipped with a complete control and data monitoring system used for many subsystems ( sections) of the boat.

In the engine room an ALBA-Engine interface that gathers signals from analog engine sensors, has been installed. The interface is actually a $\mathrm{D} \backslash \mathrm{A}$ converter and it transmits gathered data in the NMEA 2000 message format, through the web. Apart from the engine sensors, extra devices that measure propeller shaft vibration and exhaust gas temperature, were added. Every section (lighting, batteries, comfort subsystems) has been equipped with a similar transducer designed for particular types of sensors and actuators. For example the ALBA-Lighting module consists of 8 binary inputs, into which light source circuits are connected, as well as the same number of current relays switched on by the device after receiving a proper command-message from the network. The connection diagram of analog (passive) sensors with the network is shown in Fig. 6.

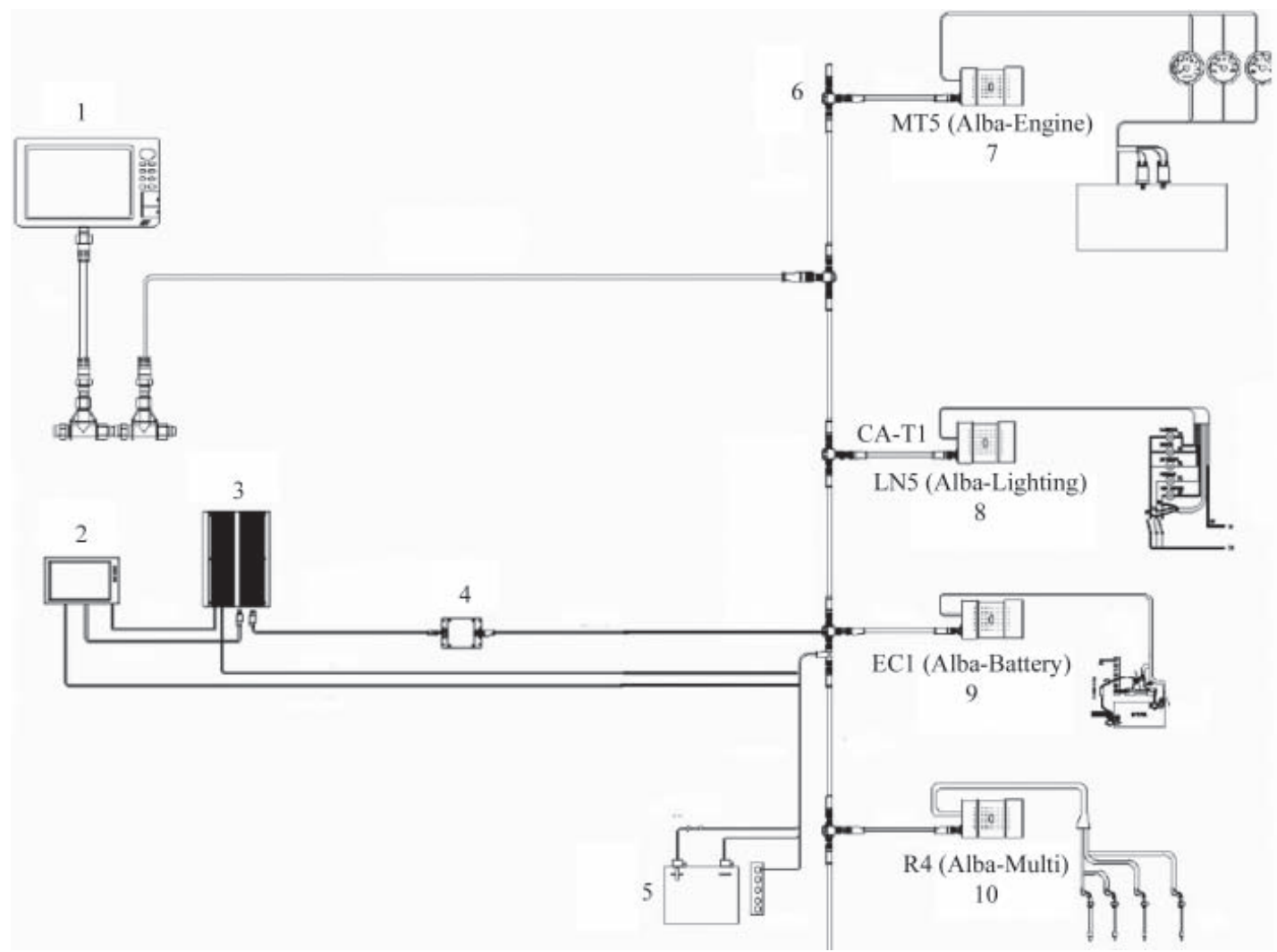

Fig. 6. A part of connection diagram of Albatross Control and Management System: 1 - multi-function display (Raymarine - G-series) at the helm station; 2 - touch screen in the navigation cabin; 3 - ALBA-CPU computer; 4 - ALBA-USB/WIFI - NMEA2000 to USB and Wireless LAN converter (the module enables connecting the onboard computer and a laptop to the ship's network); 5 - network power source; $\mathbf{6}$ - dedicated " $T$ " junction that connects the device drop cable to the main bus; 7 - ALBA-Engine -engine room controller; 8 -ALBA-lighting - light system controller; 9 - ALBA-battery - energy management; 10 - ALBA-Multi - control and data acquisition from the comfort devices. Notation: in order to simplify the picture not all system elements were shown. The rest of individual sections, converter modules and active network elements are connected similarly by a drop cable and a "T" connection ( specified in the NMEA 2000 standard) to the backbone cable

\footnotetext{
${ }^{1}$ All photos and diagrams of this section are provided by courtesy of Emmi Network [10] - the designer of the system.
} 
Apart from the passive sensors and actuators which communicate with the network by the Albatross system transducers, many active devices (connected directly to the network) have been installed on the ship. The devices are mainly navigational instruments (depth sounder, log, GPS antenna, multifunction displays etc.), dedicated to the NMEA 2000 systems. The characteristics of the standard enables a non-conflicting functioning of devices made by different manufacturers. The application of dedicated NMEA 2000 sensors for fuel, water and wastewater level measurement was a very interesting solution used on board. The whole resource management section has been built by using active elements. Figure $5 \mathrm{~b}$ presents the fuel filler used in the project. This element is prepared to function as a separate node in the network and it is plugged directly to the main bus with a drop cable.

The heart of the system is an ALBA-CPU water and shock resistant industrial computer. The unit gathers information from all nodes of the NMEA network. The management and visualization software "Albatross On Board Client" has been introduced to the computer. The access to the application is made possible through 3 terminals - a touch screen at the helm station, a similar device in the navigation cabin and through the owner's laptop (by means of wireless network)

The „Albatross On Board Client” software enables the user to freely reconfigure the monitors to customize the way the data gathered in any section, is displayed. Also, interactive fields which can serve to change parameters (like temperature inside), to choose work modes etc, can be put onto the screen. The application terminals configured to visualize engine parameters at the helm station, is shown in Fig. 7.

The down side of the picture shows the visualization of the boat lighting system. The user, by using the interactive screen, can turn the lights on or off in any part of the yacht. Obviously the potential of the system is much greater. It is possible to define alarms when the element status changes or a parameter value exceeds pre-set limit. An automatic reaction can be assigned to any event. For example, a reaction of the system for the voltage drop in one of the battery modules could be either switched to energy saving mode (the system will turn-off non-critical devices) or automatic engine/generator start-up to charge the batteries. All this can be done without the operator's intervention. The owner has the possibility to remotely monitor status of tanks and other parameters as well. Engine warm-up can be initiated in advance of getting onto the yacht etc. Thanks to the use of CAN protocol, the presented system makes it possible to apply the technology of the most exclusive glass-cockpit solutions, with just a fraction of its traditional cost.

\section{SUMMARY}

The requirements concerning the on-board communication networks are very demanding. The CAN protocol on which the NMEA 2000 network is based are capable of fulfilling them only as far as the communication between separate sensors and actuators is concerned. The low cost of components and easiness of configuration of the network elements surely contribute to maintaining the standard in this area. The procedure of removing errors in the CAN network is not adaptive hence it takes sometimes more time than in case of other protocols. Unless the very removing of errors is more important than their detecting, the cheap CAN controllers will be a better solution. The CAN has been developed for over 17 years and there is the opinion [9] that elaboration of any other solution capable of replacing it, would take as much time as that.
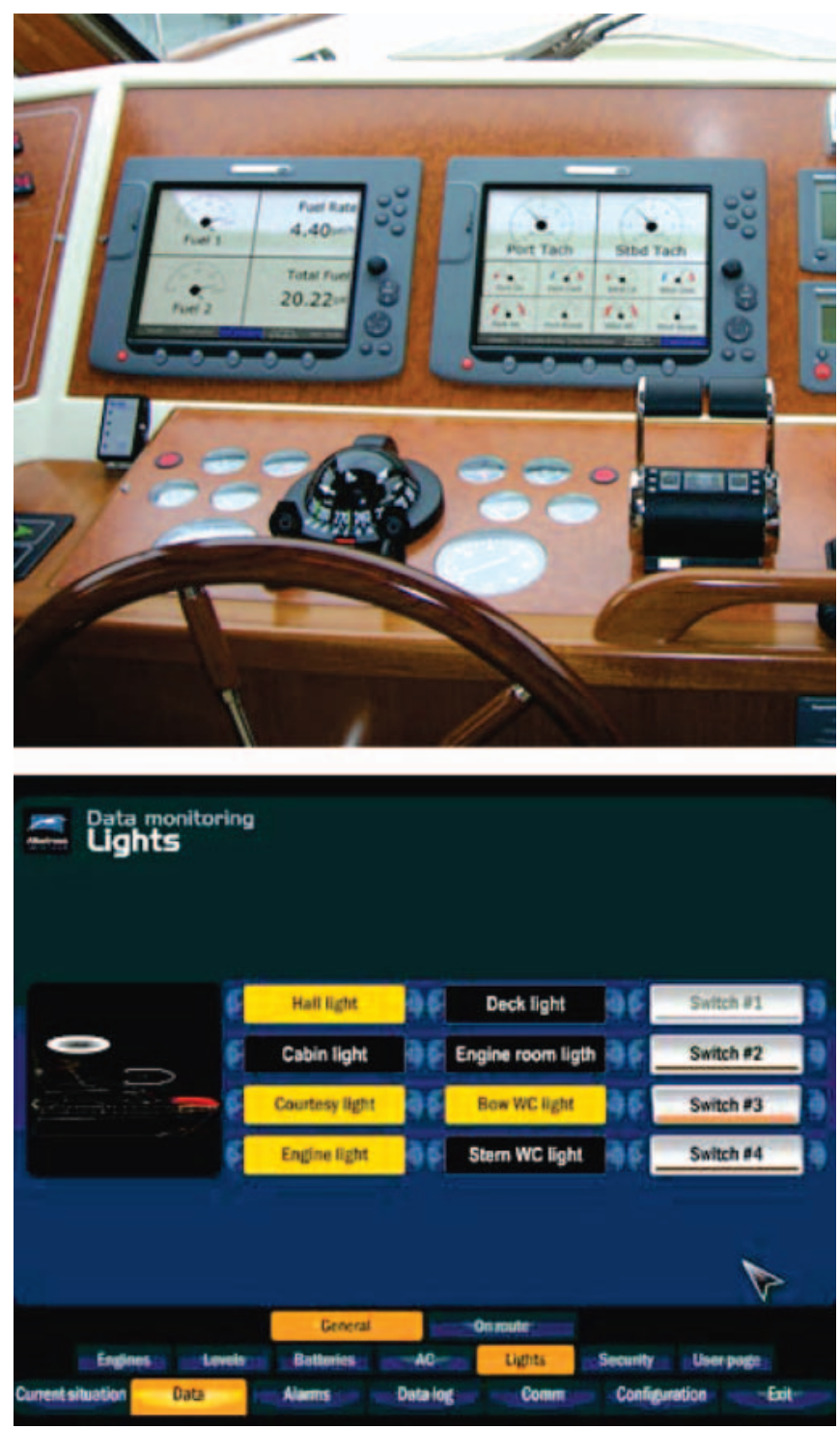

Fig. 7. The helm station in which the monitors were configured to display engine parameters. Down side the control screen of one of the sub-sections (lighting) is visible

Speed of data transfer depending on the length of the backbone cable, seems to be the main limitation for applying the CAN on board the vessels. It significantly weights in favour of the rival solution - the standardized vessel's LAN (MiTS) networks whose development in the direction of real-time systems is possible along with synchronization of clocks in all the central units. Capacity of the NMEA 2000 network can be further expanded by means of optimizing the electronic equipment as well as data transfer and form, therefore further research should be carried out in this direction.

It is worth noticing that as far as big vessels are concerned the applying of CAN systems is limited to the actuatorsensor network. Such communication system dominates on small sailing and motor vessels, where it has been implemented till now in the conventional electric control systems with the limited use of the NMEA 0183 protocol. Its low cost and possible simplification of electric installation, as well as easiness of introducing redundancy into critical systems (double main), make the new standard ideal for use on board small crafts. Also, first applications of the NMEA 2000 networks have already appeared on the motor vessels [10], however, the knowledge about the system is 
still rather not wide- spread among the designing teams. Taking into consideration the significant share of the products of Polish pleasure craft industry in their market segment, it seems advisable to promote the solution among the producers and designers, as this innovation could strengthen competitiveness of their companies. Also, to conduct further research on possible application of the NMEA 2000 standard on board the recreational vessels is advised.

\section{BIBLIOGRAPHY}

1. Simrad AS: SimNet Installation Manual 2004

2. Raymarine plc: SeaTalk Reference Manual 2007

3. ISO standard 7498-1: Information technology - Open Systems Interconnection - Basic Reference Model: The Basic Model, 1994

4. Cassidy F.: NMEA 2000 Explained - The latest word. Marine Electronics, 1999

5. Luft L. A., Anderson L., Cassidy F.: NMEA 2000 - A Digital Interface for the $21^{\text {st }}$ Century. Institute of Navigation's National Technical, 2002 San Diego, California

6. National Marine Electronics Association: NMEA 2000, version draft 4.1, 1999
7. Zimmermann W., Schmidgall R.: Bussysteme in der Fahrzeugtechnik. Vieweg \& Sohn Verlag, Wiesbaden 2007

8. Rødseth Ø.: Companion standards for integrated ship control system. SINTEF Automatic Control N-7034 Trondheim, 1995 9. http://www.raymarine.com/

10.http://www.emminet.com/

11. Törgren M.: A perspective to the design of Distributed Realtime Control Applications based on CAN. 1999

12.Anderson L., Luft L. A.: NMEA 2000 Applied. RTCM, May 2002, St Petersburg, Fl, (updated in 2006).

\section{CONTACT WITH AUTHORS}

Andrzej Piętak, Assoc. Prof. Maciej Mikulski, M. Sc.

Faculty of Technical Sciences Chair of Mechatronics Słoneczna 46 A 10-710 Olsztyn, POLAND e-mail: apietak@uwm.edu.pl e-mail: maciej.mikulski@uwm.edu.pl 